$$
\begin{array}{r}
\text { CONF- } 9605173--20 \\
\text { CONF-96062/--38 } \\
\text { SLAC-PUB-7292 } \\
\text { LBNL-39329 } \\
\text { September } 1996
\end{array}
$$

\title{
Observation, Control and Modal Analysis of Longitudinal Coupled-Bunch Instabilities in the ALS via a Digital Feedback System
}

J. D. Fox, R. Claus, H. Hindi, I. Linscott, S. Prabhakar, W. Ross, D. Teytelman*

Stanford Linear Accelerator Center, Stanford, CA 94309

A. Drago, M. Serio

INFN - Laboratori Nazionali di Frascati, P.O. Box 13, I-00044 Frascati

(Roma), Italy

J. Byrd, J. Corlett, G. Stover

Lawrence Berkeley Laboratory, 1 Cyclotron Road, Berkeley, CA 94563

\begin{abstract}
The operation of a longitudinal multi-bunch damping system using digital signal processing techniques is shown via measurements from the LBL Advanced Light Source. The feedback system (developed for use by PEP-II, ALS and DA $\Phi N E$ ) uses a parallel array of signal processors to implement a bunch by bunch feedback system for sampling rates up to $500 \mathrm{MHz}$. The programmable DSP system allows feedback control as well as accelerator diagnostics. A diagnostic technique is illustrated which uses the DSP system to excite and then damp the beam. The resulting $12 \mathrm{~ms}$ time domain transient is Fourier analyzed to provide the simultaneous measurement of growth rates and damping rates of all unstable coupled-bunch beam modes.
\end{abstract}

\section{Presented at the}

7th Beam Instrumentation Workshop (BIW 96)

Argonne National Laboratory, Argonne, IL May 6-9 1996

and Presented in abbreviated form at the

5th European Particle Accelerator Conference (EPAC 96)

Sitges, Spain June 10-14 1996 


\section{DISCLAIMER}

This report was prepared as an account of work sponsored by an agency of the United States Government. Neither the United States Government nor any agency thereof, nor any of their employees, make any warranty, express or implied, or assumes any legal liability or responsibility for the accuracy, completeness, or usefulness of any information, apparatus, product, or process disclosed, or represents that its use would not infringe privately owned rights. Reference herein to any specific commercial product, process, or service by trade name, trademark, manufacturer, or otherwise does not necessarily constitute or imply its endorsement, recommendation, or favoring by the United States Government or any agency thereof. The views and opinions of authors expressed herein do not necessarily state or reflect those of the United States Government or any agency thereof. 


\section{DISCLAMIER}

Portions of this document may be illegible in electronic image products. Images are produced from the best available original document. 


\section{System Description}

An electronic system to add damping to the longitudinal bunch motion of a storage ring requires components to measure the energy of particle bunches, a processing system to compute a correction signal from the energy errors, and a power stage to correct the energy of the particles. We have developed a damping system that uses a digital signal processing formalism to compute correction signals. Such a technique is extremely flexible, allowing many operating modes and diagnostic mmeasurements; and general purpose in that a single hardware design can be used by many accelerator facilities (the specific operating requirements of each system are implemented via software parameters). Figure 1 presents a block diagram of the processing system, which has been developed for use at the SLAC PEP-II accelerator. The heart of the signal processing is a scalable array processor (composed of commercial digital signal processing microprocessors) which provides a flexible signal processing block(1-3). The digital processing array is composed of 40 processors which provide an aggregate multiply-accumulate rate of $1.6 * 10^{9}$ operations $/ \mathrm{sec}$.

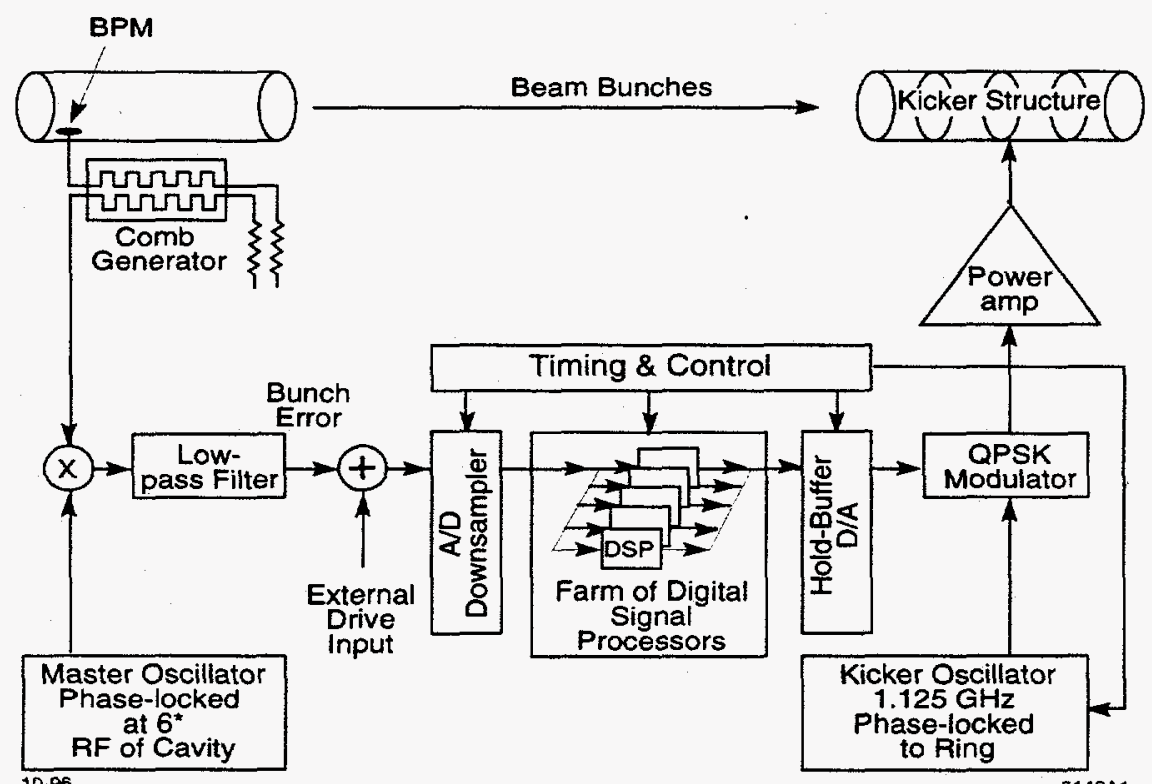

Figure ${ }^{1096}$. Block diagram of the longitudinal feedback system. The arrây $y^{81}$ of digital signal processors operate in parallel to compute correction signals on a bunch by bunch basis.

\section{Experimental Results}

The prototype longitudinal feedback system has been installed at the Advanced Light Source at Lawrence Berkeley Laboratory. The ALS machine is a third generation storage ring, with a $\mathrm{RF}$ frequency of $500 \mathrm{MHz}$ and a harmonic number of 328 . The ALS implementation requires a $500 \mathrm{MHz}$ bunch crossing and error correction rate. The ALS machine has displayed evidence 
Table 1. Parameters of Accelerator, Feedback System

\begin{tabular}{|c|l|l|} 
Parameter & Description & Value \\
\hline$E$ & Beam energy & $1.5 \mathrm{GeV}$ \\
$f_{r f}$ & RF frequency & $499.65 \mathrm{MHz}$ \\
$h$ & Harmonic number & 328 \\
$f_{r e v}$ & Revolution frequency & $1.5233 \mathrm{MHz}$ \\
$\alpha$ & Momentum compaction factor & $1.594 \mathrm{e}-3$ \\
$I_{0}$ & Operating current & $400 \mathrm{~mA}$ \\
$f_{s}$ & Synchrotron frequency & $11 \mathrm{kHz}$ \\
- & Bunch sampling rate & $499.65 \mathrm{MHz}$ \\
- & Downsampling factor & $21-31$ \\
$N$ & Digital Filter Length & 6 \\
- & Feedback loop gain & $6-28 \mathrm{~dB}$ \\
- & Feedback output power & $200 \mathrm{~W}$ \\
- & Output amplifier bandwidth & $1-2 \mathrm{GHz}$ \\
\hline
\end{tabular}

of strong longitudinal instabilities since commissioning in April 1993(4). Table 1 lists relevant parameters for the accelerator and the feedback system. The system was commissioned for routine operation in September 1995, and has been used for production running of the light source at both 1.5 and 1.9 $\mathrm{GeV}$ energies. Operated in conjuction with an all-mode transverse feedback system $(5)$ the feedback systems have demonstrated increased intensity and reduced linewidths of the emitted higher order undulator radiation. The feedback systems suppress coupled-bunch instabilities up to the full ( $400 \mathrm{~mA})$ operating current of the storage ring.

The feedback error processing is implemented in a bunch-by-bunch discrete time system, in which the error signal for a particular bunch is computed using several past measurements of only that bunch. The algorithm used is a Finite Impulse Response ( FIR) discrete time filter(6)

$$
u_{i}(n)=\sum_{k=1}^{N} h(k) \phi_{i}(n-k) .
$$

with coefficients selected to provide maximum gain at the synchrotron frequency, and with the filter phase shift adjusted to provide net negative feedback at the synchrotron frequency. The action of the parallel processing array, and the parallel computation of all the populated bunches creates an all-mode global transfer function which folds the single bunch transfer function around all the revolution harmonics in the spectrum of the bunch motion.

The digital processing scheme allows the computation of linear or non-linear ( saturated) correction signals. One interesting feature of the programmable system is the capability to record the bunch motion as the feedback system operates.(7) Such digital data records provide a very powerful diagnostic capa- 
bility to observe and quantify the motion of the particles in the storage ring. In particular, observing the motion as the feedback system is turned off displays the growth rates of the unstable modes, while recording the motion when the feedback signals are turned back on reveals the net damping in the system. Frequency domain information can be computed from these time-domain data sets via use of Fourier transform techniques.

An example measurement using this grow-damp technique is illustrated in Figure 2. In this experiment the beam is initially stable under the action of the feedback system. Under software control the feedback gain is set to zero, and after a holdoff interval the DSP processors start recording the bunch motion. For $t_{\text {holdoff }}<t<t_{\text {on }}$ the growing bunch oscillations are recorded, and at $t_{\text {on }}$ the feedback gain is restored to the operating value. The motion ( now a damping transient) continues to be recorded until $t_{\max }$, at which time the DSP's stop recording but continue computing the feedback signal (controlling the beam). The data records are stored in a dual-port memory which is accessible to an external processor (the total record length is 960 samples of each of 324 bunches). The recorded data is processed off-line. After the data array is read, the DSP processors can be triggered again to record another transient.

Figure 2a shows the envelopes of the synchrotron oscillations of each of 328 bunches for such a transient. We see a complicated growth of motion up to $6 \mathrm{~ms}$, followed by damping of the motion. In this representation the phase relationship of the individual bunch oscillations is not obvious. If the data is arranged in a 2 dimensional array of bunch \# vs. sample number, each row ( containing the oscillation co-ordinate of each of 328 bunches sampled on a single turn) ${ }^{*}$ can by fourier transformed to reveal modes of oscillation of the bunches. This Fourier transform is computed for each sampling time ( turn number) in the array. The resulting data is displayed in Figure $2 \mathrm{~b}$, which shows the presence of two modes of oscillation in the transient. $\dagger$ The growth rates and damping rates for each mode can be found from this data set via the numeric fitting of exponential curves to the data, as shown in figure $2 c$,e. Figure $2 \mathrm{~d}, \mathrm{f}$ present the effective growth rate and damping rate vs. mode number - we see here the action of the feedback system in turning the net growth rate from positive to negative.

This figure also reveals the gain margin in the damping system. The instability growth rate per mode is due to a net effective impedance ( found by a

\footnotetext{
${ }^{*}$ Due to the action of the downsampled processing, the data for the various bunches are actually sampled on different turns corresponding to the sampling pattern and downsampling factor. In the post-processing an interpolating filter is used on each bunch's column in the raw data array to compute the oscillation co-ordinates over the non-sampled turns, effectively time-aligning all the bunch co-ordinate data before the Fourier transforms are taken.

tThe Fourier transform of a spatial record of length $\mathrm{N}$ is decomposed into $\mathrm{N} / 2$ spatial frequencies - thus in this representation the 328 modes of the bunch system is folded into the 164 modes in the turn Fourier transform. Each computed "mode" actually contains two true beam modes corresponding to the upper and lower synchrotron sidebands around a particular revolution harmonic. $(9,10)$
} 

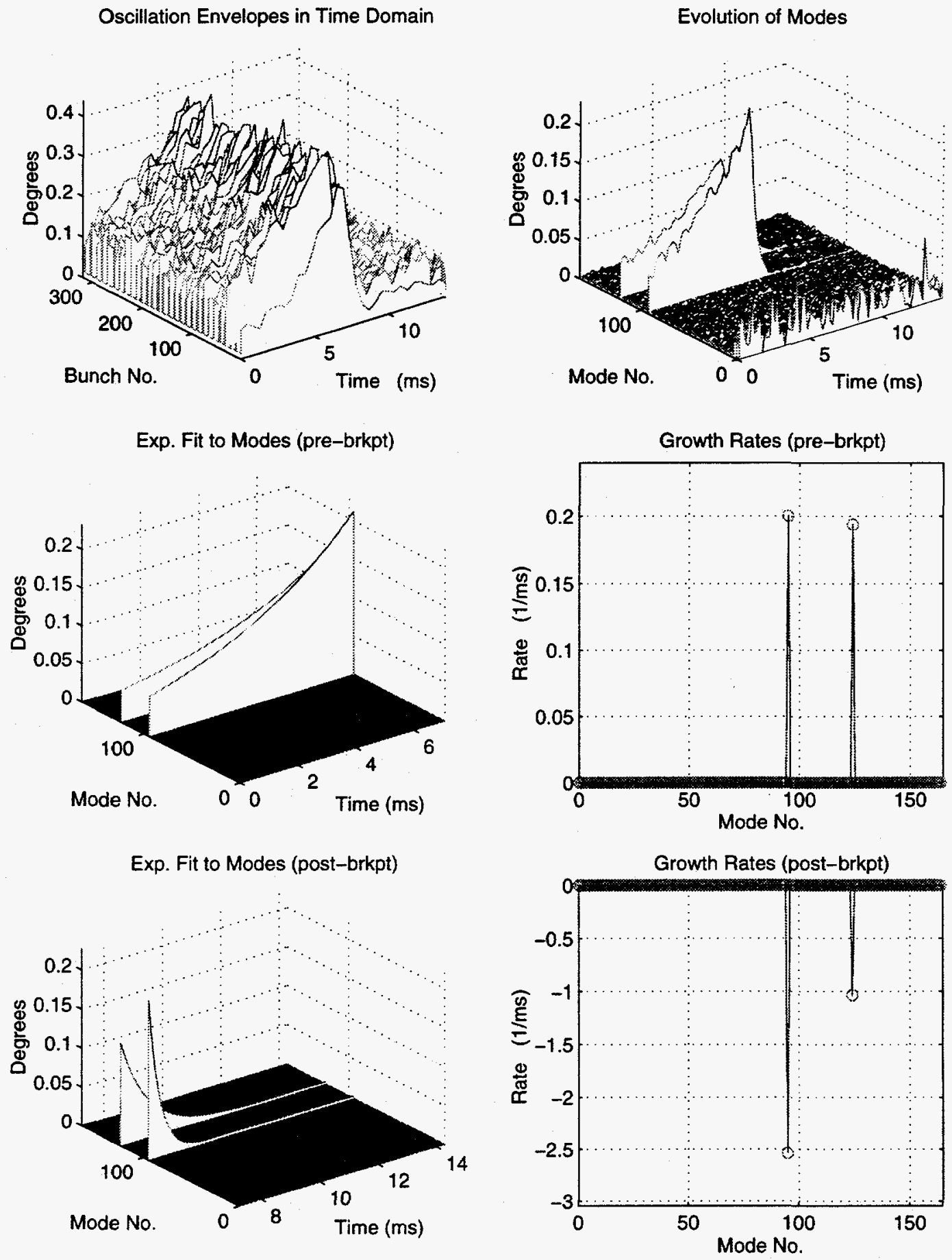

tes06962105: $10=118.4 \mathrm{~mA}$, Dsamp=22, Shit Gain= A, Nbun=320, Gain $1=0$. Gain2 $=1$, Phase1 $=30$, Phase $2=-140$, Brkpt $=496$, Calib 24 cnts/mAmdeg.

Figure 2. 
summation over revolution harmonics)(8)

$$
\begin{aligned}
1 / \tau_{l} & =\frac{I_{0} f_{r f} \alpha}{2(E / e) Q_{s}} \operatorname{Real}\left(Z_{l}^{e f f}\right) \\
Z_{l}^{e f f} & =\sum_{p=-\infty}^{p=+\infty} \frac{\omega_{p}}{\omega_{r f}} \exp \left(-\omega_{p}^{2} \sigma_{\tau}^{2}\right) Z\left(\omega_{p}\right) \\
\omega_{p} & =\left(p N+l+Q_{s}\right) \omega_{r e v},
\end{aligned}
$$

where $\tau_{l}$ is the growth time of mode $l$ due to the ring impedance, $I_{0}$ is the beam current, $Z(\omega)$ is the total ring impedance, $e$ is the charge of an electron, $Q_{s}$ is the synchrotron tune $\left(f_{s} / f_{r e v}\right), \sigma_{\tau}$ is the rms time-of-arrival variation of particles within a bunch, and $N$ is the number of bunches (see Table 1 for more symbol definitions).

The action of feedback system provides a damping rate which is proportional to the gain at that mode; the net response of the system is determined by the difference between the two ( natural growth and feedback damping) rates

$$
\begin{aligned}
1 / \tau^{f b} & =\frac{f_{r f} \alpha}{2(E / e) Q_{s}} G_{f b} \\
1 / \tau_{l}^{\text {net }} & =1 / \tau_{l}-1 / \tau^{f b}
\end{aligned}
$$

which is plotted per excited mode in figure $2 \mathrm{f}$.

This approach directly measures the unstable modes of the ring - with a variant of this technique it is possible to measure damping/growth rates for naturally stable modes. For this measurement a narrowband excitation at the desired mode frequency is injected into the feedback system at the error summing node ( see figure 1). The feedback system passes this excitation signal onto the beam via the power stage and kicker, and the bunch motion at that specific frequency is excited ( a single mode of the beam). If the excitation and feedback is turned off, the excited mode decays (or grows!), and as the feedback is turned on the net damping rate increases. The grow-damp transient can be recorded and processed just as for the self-excited case. Figure $3 a, b, c, d, e, f$ shows such a measurement for an excitation of mode \# 61. The figure reveals a natural damping rate of the mode $(-0.011 / \mathrm{ms})$ as well as the action of the feedback system to provide a net damping rate of $-0.91 / \mathrm{ms}$. If this measurement is repeated for several modes (or an excitation is applied which excites several modes) the effective gain of the feedback system vs. mode can be determined. Measurement of such a frequency dependent effective gain is a useful system check, and is particularly helpful in the determination of the frequency responses of the wideband power amplifier and kicker stages.

\section{Summary}

The programmable nature of the DSP processing system allows measurements of beam motion as well as computation of feedback control signals. The 

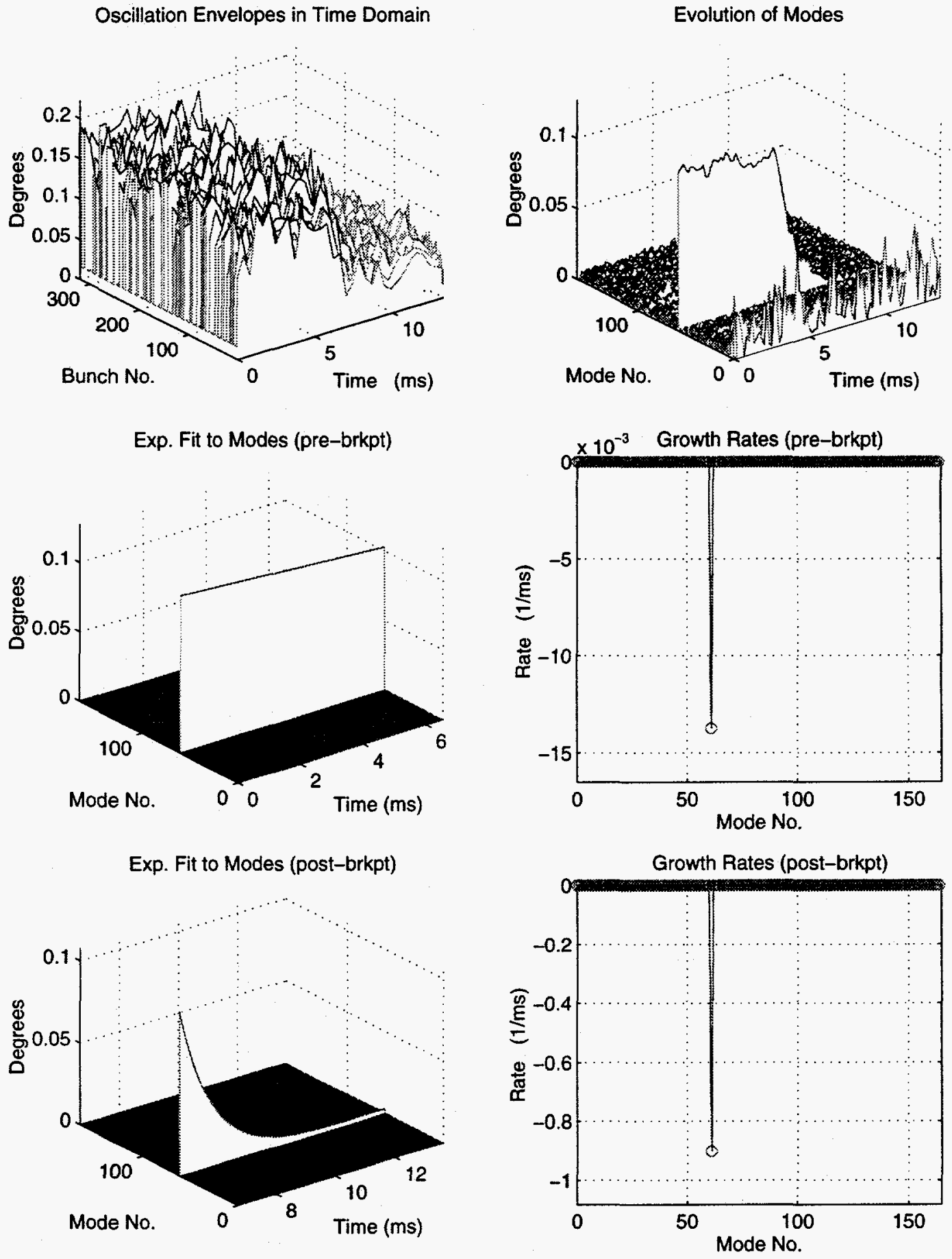

teb2796/2104: 10=147.9ma, Dsamp $=21$, Shift Gairs 3 , Nbun= 320, Gain1 $=0$, Gain $2=1$, Phase $=-140$, Phase $=-140$, Brkpt= 480, Calb $=24$ chtsimA-deg.

Figure 3. 
time domain transient techniques illustrated require only a few ms of beam motion and are essentially invisible to users of the storage ring. The information obtained from the analysis of the time domain data reveals growth rates and damping rates of beam modes, and is very useful in adjusting the feedback system or as a quick check on its operation.

The time domain techniques, in conjunction with off-line FFT analysis, are complementary to narrowband detection in the frequency domain, in that they allow the quantification of many unstable modes in a single non-destructive transient, and do not require repetitive narrowband steady state swept frequency domain measurements of each potentially unstable mode. The essential advantage of the time domain technique is speed - successive narrowband sweeps of each possibly unstable mode can reveal the same information. However, for an accelerator with potentially hundreds or thousands of unstable modes and constantly drifting parameters ( such as cavity temperature or cavity tuning) the speed advantage makes the time-domain techniques very useful as accelerator diagnostics.

\section{Acknowledgments}

The authors thank J. Hoeflich, J. Olsen, G. Oxoby of SLAC and G. Lambertson of LBL for numerous thoughtful discussions and direct contributions of technical expertise. We also thank Boni Cordova-Gramaldi of SLAC for her patient fabrication of electronic components.

\section{Figure Captions}

[2] a)Plot of the oscillation envelopes of 328 bunches in the ALS over a 12 ms time interval. The feedback system is on for $t<0$ and $t>6 \mathrm{~ms}$, and off for $0<t<6 \mathrm{~ms}$. Unstable bunch motion grows during the feedback off interval. b) is a turn by turn FFT of the bunch data, revealing two unstable modes ( mode -95 and -124). c) is an exponential fit to first $6 \mathrm{~ms}$ of b). d) is the fitted growth rate vs. mode \#. e) shows the fit damping exponentials ( last $6 \mathrm{~ms}$ of b). $f$ ) is the net damping rate vs. mode \#( showing the action of the feedback system).

[3]Plot in the same manner as figure \#2, but with a narrowband excitation of mode \#61 applied to the summing node of the feedback system. The natural damping rate is seen in c) and d), the damping with feedback in e) and f)

\section{REFERENCES}

1. J. Fox et al. "Feedback Control of Coupled-Bunch Instabilities," Proc. Particle Accelerator Conference, 1993.

2. R. Claus et al, "Software Architecture of the Longitudinal Feedback System for PEP-II, ALS and DA $\Phi$ NE," Proc. Particle Accelerator Conference, Dallas, 1995. 
3. D. Teytelman, et al, "Operation and Performance of the PEP-II Prototype Longitudinal Damping System at the ALS," Proc. Particle Accelerator Conference, 1995

4. A. Jackson, et al. "Commissioning and Performance of the Advanced Light Source," Proc. Particle Accelerator Conference, 1993

5. W. Barry, et al, "Commissioning and Operation of the ALS Transverse Coupled-Bunch Feedback System", Proc. Particle Accelerator Conference, Dallas, 1995

6. A. Oppenheim, R. Schafer, Discrete-Time Signal Processing, Prentice Hall, 1989.

7. D. Teytelman, et al, "Feedback Control and Beam Diagnostic Algorithms for a Multi-processor DSP System," Proc. Beam Instrumentation Workshop, 1996 ( this conference)

8. M. Zisman et al, Lawrence Berkeley Lab. Report No. LBL-21270.

9. F. Sacherer, "A Longitudinal Stability Criterion for Bunched Beams," IEEE Trans. Nucl. Sci., NS-20-3, p. 825 (1973)

10. J.M. Wang, "Modes of Storage Ring Coherent Instabilities," AIP Conf. Proc. 153 (1985) 